Stud. Univ. Babeş-Bolyai Math. 64(2019), No. 3, 339-347

DOI: http://dx.doi.org/10.24193/subbmath.2019.3.05

\title{
Choquet boundary for some subspaces of continuous functions
}

\author{
Laura Hodiş and Alexandra Măduţa
}

Dedicated to Professor Heiner Gonska on the occasion of his 70th anniversary.

\begin{abstract}
We investigate the Choquet boundary for subspaces of parabolic functions and for linearly separating subspaces of continuous functions. The relation of the Choquet boundary with the set of peak points is also investigated.
\end{abstract}

Mathematics Subject Classification (2010): 46A55.

Keywords: Choquet boundary, parabolic functions, linearly separating subspaces, peak points.

\section{Introduction}

It is well known that Choquet Theory provides a unified approach to integral representations in several areas of mathematics: potential theory, probability, function algebras, operator theory, group representations, ergodic theory (see, e.g., $[1-3,10,14])$. Particularly, the Choquet boundary is an essential tool in Korovkin approximation theory (see, e.g., $[2,3,5,9,11,12]$ ).

In this paper we are concerned with the Choquet boundary for subspaces of parabolic functions and linearly separating subspaces of continuous functions. For other results concerning boundaries, parabolic functions, linearly separating subspaces see, e.g., $[1-4,6,7,10,12,14]$ and the references therein.

Section 2 is devoted to subspaces of parabolic functions. We recall some known results about the Choquet boundary of such a subspace, motivated by their relations with Korovkin theory. The relation between the Choquet boundary and the set of peak points is also investigated.

In Section 3 we study the Choquet boundary for linearly separating subspaces. Important results in this direction were obtained in $[6,13]$, and the references therein. Our main result is Theorem 3.1. We start with Proposition 48 from [13] and add a supplementary hypothesis; then we construct an example showing that without this hypothesis the conclusion in not generally true. 
Throughout the paper we use the following definitions and notations.

For other definitions and notations see, e.g., [1-3], [10].

For a compact Hausdorff space $X$, let $C(X)$ denote the Banach space of realvalued continuous functions on $X$, equipped with the supremum norm. Let $S$ be a subset of $C(X)$.

A subset $B$ of $X$ is called a boundary for $S$ if for each $f \in S$ there exists $b \in B$ such that $f(b)=\min _{X} f$.

Let $M(X)$ be the space of all Radon measures on $X$ and $M_{+}(X)$ the set of all Radon positive measures on $X$. Let

$$
M_{+}^{1}(X)=\left\{\mu \in M_{+}(X): \mu(1)=1\right\} .
$$

For $x$ in $X$ let $e_{x}$ be the corresponding Dirac measure on $X$.

If $S$ is a subset of $C(X)$ and $\mu, \gamma$ are in $M^{+}(X)$, we write $\mu \prec_{S} \gamma$ (or, simply, $\mu \prec \gamma$ ) if $\mu(s) \leq \gamma(s)$ for all $s$ in $S$.

The Choquet boundary of $X$ with respect to $S$ is the set

$$
C h(S)=\left\{x \in X: \mu \in M_{+}^{1}(X), \mu \prec e_{x} \Rightarrow \mu=e_{x}\right\} .
$$

If $S$ separates the points of $X$, then $C h(S)$ is a boundary for $S$ (see [4]).

Let us consider the set of peak points with respect to $S$ (see [1, p. 39]):

$P(S)=\{x \in X: \exists f \in S, f(x)<f(y)$ for all $y \in X \backslash\{x\}\}$.

It is easily seen that $P(S) \subset C h(S)$.

If $S$ is a linear subspace of $C(X)$, then

$$
C h(S)=\left\{x \in X: \mu \in M_{+}^{1}(X), \mu_{\left.\right|_{S}}=e_{\left.x\right|_{S}} \Rightarrow \mu=e_{x}\right\} .
$$

\section{The Choquet boundary for subspaces of parabolic functions}

Let $E$ be a locally convex Hausdorff space over $\mathbb{R}$, and $K$ a compact metrizable convex subset of $E$. We denote by $A(K)$ the set of all continuous real-valued affine functions on $K$ and by ex $K$ the set of all extreme points of $K$.

Theorem 2.1. ([1, Proposition I.4.3]) The Choquet boundary of the subspace $A(K)$ coincides with ex $K$.

We shall see that the Choquet boundary of the linear subspace of $C(K)$ generated by $A(K)$ and $f \in C(K)$, coincides with $K$.

Let $f \in C(K)$ be convex. Then, it is known that $f$ has a right Gateaux derivative, given by

$$
D f(x ; y)=\lim _{t \downarrow 0} \frac{f(x+t y)-f(x)}{t}=\inf _{t>0} \frac{f(x+t y)-f(x)}{t}
$$

for all $x, y$ such that $x \in K, x+y \in K$.

We will say that $f$ is smooth provided that for all $x \in K$ the mapping

$$
a_{x}: K \rightarrow \mathbb{R}, \quad a_{x}(y)=D f(x ; y-x) \text { is in } A(K) .
$$

Now let $f \in C(K)$ be strictly convex. Note that such a function exists since $K$ is metrizable (see [8]). 
Let $S(f)$ be the subspace of $C(K)$ spanned by $A(K)$ and $f$. The functions belonging to $S(f)$ are called parabolic functions. These subspaces were studied by C.A. Micchelli [9]. In particular, in [9, Proposition 3.1] he proved that, under the assumption that $f$ is strictly convex and smooth, then $e_{x} \in U(S(f))$ for all $x \in K$, where

$$
U(S(f))=\left\{\mu \in M_{+}^{1}(K): \gamma \in M_{+}^{1}(K), \gamma_{\left.\right|_{S(f)}}=\mu_{\left.\right|_{S(f)}} \Rightarrow \gamma=\mu\right\} .
$$

This implies

Theorem 2.2. If $f \in C(K)$ is strictly convex and smooth, then $C h(S(f))=K$.

In $[11$, Proposition 2] it was shown that the results due to C.A. Micchelli remain true if we omit the hypothesis that $f$ is smooth. Then we get the result.

Theorem 2.3. If $f \in C(K)$ is strictly convex, then $C h(S(f))=K$.

From this it follows that if $f \in C(K)$ is strictly convex, then the subspace of parabolic functions $S(f)$ is a Korovkin subspace of $C(K)$. This result was proved in [5] in the case when $K$ is a compact convex subset of $\mathbb{R}^{n}$ and in [9] in the general case under the hypothesis that $f$ is smooth.

As far as the peak point set of $S(f)$ is concerned, we state the following result.

Theorem 2.4. Let $K$ be metrizable, and $f \in C(K)$ be strictly convex and smooth. Then $P(S(f))=K$.

Proof. Let $x \in K$ and consider the function

$$
s: K \rightarrow \mathbb{R}, s(y)=f(y)-f(x)-a_{x}(y) \text { for all } y \in K .
$$

Then $s \in S(f), s(x)=0, s(y)>0$ for all $y \in K \backslash\{x\}$. Thus $x \in P(S(f))$.

Remark 2.5. If $f \in C(K)$ is strictly convex but is not smooth, it is possible to have $P(S(f)) \neq K$.

This is shown in:

Example 2.6. Let $K=[-1,1] \times[-1,1]$ and let

$$
f: K \rightarrow \mathbb{R}, \quad f(x, y)=x^{2}+y^{2}-\sqrt{1-y^{2}} \text { for all }(x, y) \in K .
$$

Then $f$ is strictly convex on $K$. By Theorem 2.3 we have $C h(S(f))=K$. But $P(S(f))=K \backslash\{(x, \pm 1):|x|<1\}$.

\section{The Choquet boundary for linearly separating subspaces of $C(X)$}

Let $H$ be a linear subspace of $C(X)$ which separates the points of $X . H^{*}$ denotes the dual of $H$, equipped with the weak * topology.

Let us consider the map

$$
\Phi: X \rightarrow H^{*}, \Phi(x)(h)=h(x) \quad \text { for all } \quad x \in X, \quad h \in H .
$$

$\Phi$ is easily seen to be a homeomorphism between $X$ and $\Phi(X)$. Now set

$$
Y=\overline{c o}(\Phi(X)) \text {. }
$$


Then $Y$ is a compact convex subset of $H^{*}$. We have (see $\left.[10,13]\right)$

$$
\operatorname{ex} Y=\Phi(C h(H)) \text {. }
$$

Let us denote

$$
\begin{gathered}
H^{+}=\{h \in H: h \geq 0\} . \\
\left(H^{*}\right)^{+}=\left\{h^{*} \in H^{*}: h^{*}(h) \geq 0 \text { for all } h \in H^{+}\right\} .
\end{gathered}
$$

Proposition 3.1. (see, [Prop. 46, 13]). Let us consider the following assertions:

(1) $H=H^{+}-H^{+}$,

(2) $\left(H^{*}\right)^{+} \cap\left(-\left(H^{*}\right)^{+}\right)=\{0\}$,

(3) $0 \notin \Phi(X)$,

(4) $0 \notin Y$,

(5) For all $x \in X$ there exists $h \in H$ such that $h(x) \neq 0$,

(6) There exists $h_{0} \in H$ such that $h_{0}>0$,

(7) $\left(H^{*}\right)^{+}$has a compact base.

Then we have:

$(3) \Longleftrightarrow(5) \Longleftarrow(4) \Longleftrightarrow(6) \Longrightarrow(1) \Longrightarrow(2),(6) \Longrightarrow(7),(2)$ and $(5) \Longleftrightarrow(6)$.

Let $F$ be a subset of $C(X)$ and set

$$
\partial(F)=\left\{x \in X: \mu \in M_{+}(X), \mu \prec_{F} e_{x} \Longrightarrow \mu=e_{x}\right\} .
$$

We need the following lemma.

Lemma 3.2. If $F$ is a subset of $C(X)$, the following properties hold:

(i) $\partial(F)=\partial(g F)$ for all $g \in C(X), g>0$.

(ii) Suppose that there exists $f_{0} \in F, f_{0}>0$ and for all $x \in X$ there exists $f \in F$ such that $f(x)<0$.

Then $\partial(F)=\cap\{C h(g F): g \in C(X), g>0\}$.

(iii) If there exists $f_{0} \in F$ such that $f_{0}>0$ and $-f_{0} \in F$, then $\partial(F)=C h\left(\frac{F}{f_{0}}\right)$.

Proof. (i) Fix $g \in C(X), g>0$ and $y \in \partial(F)$. Let $\mu \in M_{+}(X)$ be such that $\mu \prec_{g F} e_{y}$. Then

$$
\int_{X} f(x) \frac{g(x)}{g(y)} d \mu(x) \leq f(y) \quad \text { for all } \quad f \in F .
$$

Let us define $\gamma \in M_{+}(X)$ by

$$
d \gamma(x)=\frac{g(x)}{g(y)} d \mu(x) .
$$

From (3.2) it follows that $\gamma \prec_{F} e_{y}$, and hence

$$
\gamma=e_{y}
$$

Let now $t \in C(X)$, and set

$$
h=\frac{g(y)}{g} t .
$$

From (3.3) we obtain $\gamma(h)=h(y)$, i.e. $\mu(t)=t(y)$ for all $t \in C(X)$. Thus $\mu=e_{y}$. This means that $y$ belongs to $\partial(g F)$. So we have $\partial(F) \subset \partial(g F)$.

Now $\partial(g F) \subset \partial\left(\frac{1}{g} g F\right)=\partial(F)$, i.e. $\partial(F)=\partial(g F)$. 
(ii) We have $\partial(F)=\partial(g F) \subset C h(g F)$ for all $g \in C(X), g>0$. This yields

$$
\partial(F) \subset \cap\{C h(g F): g \in C(X), g>0\} .
$$

Let now $x \in X, x \notin \partial(F)$. Then there exists $\mu \in M_{+}(X)$ such that

$$
\begin{gathered}
\mu \prec_{F} e_{x}, \\
\mu \neq e_{x} .
\end{gathered}
$$

From hypothesis there exists $f \in F$ such that $f(x)<0$.

Then (3.4) implies $\mu(f) \leq f(x)<0$. So

$$
\mu \neq 0 \text {. }
$$

From (3.5) and (3.6) we deduce that there exists a compact subset $K$ of $X$ such that $x \notin K$,

$$
\mu(K)>0 \text {. }
$$

From Urysohn's Lemma we see that there exists a continuous function $k: X \longrightarrow[0,1]$ such that

$$
k(x)=0,\left.\quad k\right|_{K}=1 .
$$

Then (3.7) and (3.8) imply

$$
\mu(k)>0 .
$$

From hypothesis there exists $f_{0} \in F$ such that $f_{0}>0$. By (3.4) we have

$$
\mu\left(f_{0}\right) \leq f_{0}(x) .
$$

Let us consider the function $v \in C(X)$ given by

$$
v=f_{0}+\frac{f_{0}(x)-\mu\left(f_{0}\right)}{\mu(k)} k .
$$

We have

$$
v>0, \quad \mu(v)=v(x) .
$$

Now we define $\gamma \in M_{+}(X)$ by

$$
d \gamma(y)=\frac{v(y)}{v(x)} d \mu(y) .
$$

From (3.9) we deduce $\gamma(1)=1$; thus

$$
\gamma \in M_{+}^{1}(X)
$$

Let $f$ be arbitrarily chosen in $F$. Then we have

$$
\gamma\left(\frac{1}{v} f\right)=\frac{1}{v(x)} \mu(f) \leq \frac{1}{v(x)} f(x) .
$$

This implies

$$
\gamma \prec \frac{1}{v} F e_{x} .
$$

Suppose now that $\gamma=e_{x}$, i.e. $\gamma(g)=g(x)$ for all $g \in C(X)$. Let $t$ be arbitrarily chosen in $C(X)$. Let us denote

$$
g=\frac{v(x)}{v} t
$$


From $\gamma(g)=g(x)$ and from (3.10) we deduce $\mu(t)=t(x)$. This means that $\mu=e_{x}$, which contradicts (3.5). Thus we have

$$
\gamma \neq e_{x}
$$

Now (3.11), (3.12) and (3.13) imply

$$
x \notin C h\left(\frac{1}{v} F\right) .
$$

So $x \notin \cap\{C h(g F): g \in C(X), g>0\}$. This completes the proof of (ii).

(iii) Let $f_{0} \in F$ be such that $f_{0}>0$ and $-f_{0} \in F$. Then the constant functions 1 and -1 belong to $\frac{F}{f_{0}}$; hence $\partial\left(\frac{F}{f_{0}}\right)=C h\left(\frac{F}{f_{0}}\right)$. From (i) we deduce $\partial(F)=\partial\left(\frac{F}{f_{0}}\right)$ and so $\partial(F)=C h\left(\frac{F}{f_{0}}\right)$.

Thus Lemma 3.1 is completely proved.

In what follows we need the following definition. A subset $F$ of $C(X)$ is called linearly separating (see [6, p. 55]) if for all $x, y \in X, x \neq y$ there exist $f, g \in F$ such that

$$
\left|\begin{array}{ll}
f(x) & f(y) \\
g(x) & g(y)
\end{array}\right| \neq 0 .
$$

Remark 3.3. It is easily seen that $F$ is linearly separating if and only if for all $x, y \in$ $X, x \neq y$, and for all $c \in \mathbb{R}$ there exists $f \in F$ such that $f(x) \neq c f(y)$ (see [13]).

Remark 3.4. If $F$ separates the points of $X$ and $f+1$ belongs to $F$ for all $f \in F$, then $F$ is linearly separating.

Remark 3.5. If $F$ is linearly separating and $h \in C(X), h(x) \neq 0$ for all $x \in X$, then the set $h F$ is linearly separating.

Remark 3.6. A linear subspace $H$ of $C(X)$ is linearly separating if and only if for all $x, y \in X, x \neq y$, we have $\Phi(x) \neq 0$ and $\Phi(y)$ does not belong to the line generated in $H^{*}$ by 0 and $\Phi(x)$.

Remark 3.7. Let $H$ be a linear subspace of $C[0,1], \operatorname{dim} H=2$. Then $H$ is linearly separating if and only if $H$ is a Tchebycheff subspace. If $H$ is linearly separating, then there exists $h_{0} \in H$ such that $h_{0}>0$.

The following result is essentially contained in [Proposition 48, 13]. Here we introduce at $3^{0}$ the additional hypothesis that there exists $h_{0} \in H, h_{0}>0$. We shall construct an example in which, without this hypothesis, $3^{0}$ does not hold, that is

$$
\emptyset=\partial(H) \varsubsetneqq \cap\{C h(f H): f \in C(X), f>0\} \varsubsetneqq C h(H) .
$$

Theorem 3.8. Let $H$ be a linear subspace of $C(X)$. Then:

$1^{0} \partial(H) \subset C h(H)$. If $H$ contains the constant functions, then $\partial(H)=C h(H)$.

$2^{0} \partial(H)=\partial(f H)$ for all $f \in C(X), f>0$.

$3^{0}$ If there exists $h_{0} \in H, h_{0}>0$, then

$$
\partial(H)=\cap\{C h(f H): f \in C(X), f>0\}=C h\left(\frac{H}{h_{0}}\right) .
$$


$4^{0}$ If $H$ is linearly separating, then the following assertions are equivalent:

a) $\partial(H) \neq \emptyset$,

b) there exists $h_{0} \in H, h_{0}>0$,

c) $H=H^{+}-H^{+}$,

d) $\left(H^{*}\right)^{+} \cap\left(-\left(H^{*}\right)^{+}\right)=\{0\}$.

Proof. $1^{0}$ is obvious.

$2^{0}$ and $3^{0}$ follow from Lemma 3.1.

$4^{0}$ is a consequence of Proposition 3.1.

Example 3.9. Let $X=[-2,2]$. Consider the functions $h_{1}, h_{2}, h_{3}$, belonging to $C[-2,2]$ and defined for all $x \in[-2,2]$ as

$$
\begin{aligned}
& h_{1}(x)=-\frac{1}{2} x, \quad h_{2}(x)=1-|x|, \\
& h_{3}(x)=\left\{\begin{array}{lll}
1-|x+1|, & \text { if } \quad x \in[-2,0], \\
1-|x-1|, & \text { if } \quad x \in(0,2] .
\end{array}\right.
\end{aligned}
$$

Let us denote by $H$ the linear subspace of $C[-2,2]$ generated by $h_{1}, h_{2}, h_{3}$.

We identify the functional $\varphi \in H^{*}$ with the vector $\left(\varphi\left(h_{1}\right), \varphi\left(h_{2}\right), \varphi\left(h_{3}\right)\right)$; so, we identify $H^{*}$ with $R^{3}$.

$\Phi([-2,2])$ is the following curve in $R^{3}$ :

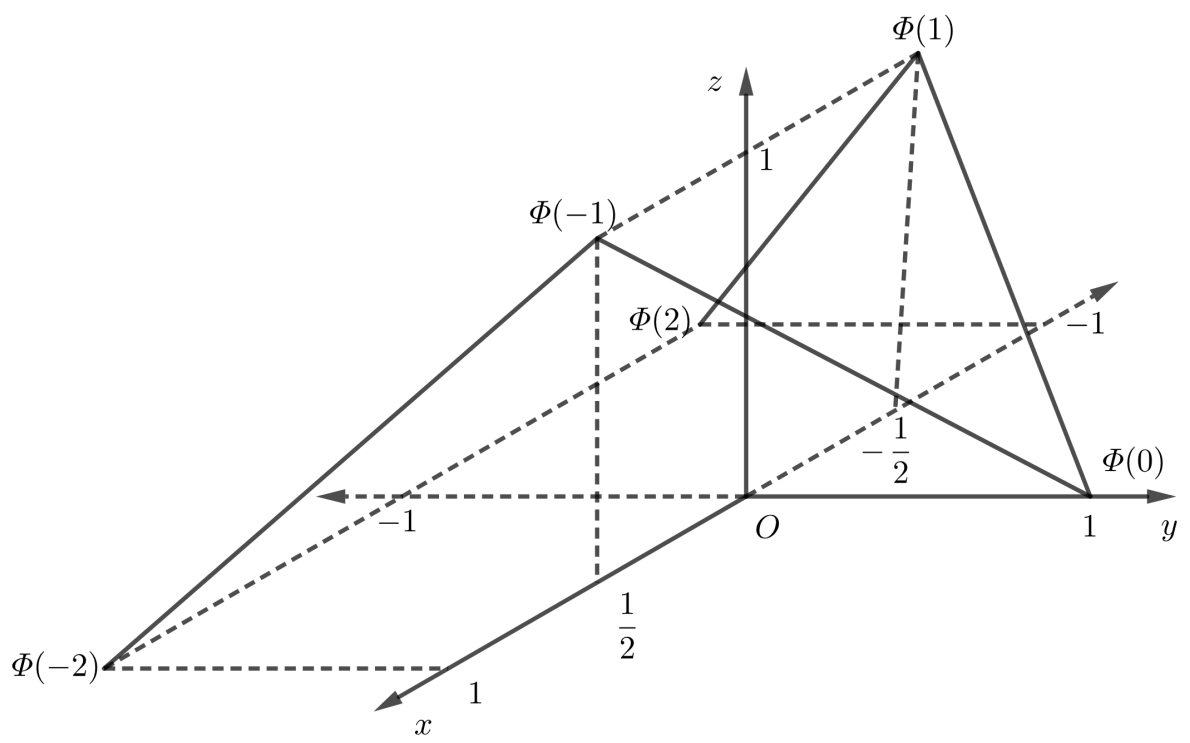


From Remark 3.6 we have that $H$ is linearly separating. Since $0 \in Y=\overline{c o}(\Phi([-2,2]))$, from Proposition 3.1 we deduce that $H$ does not contain strictly positive functions (this fact can be easily proved directly).

By Theorem 3.1 we have

$$
\partial(H)=\emptyset
$$

From (3.1) we deduce

$$
C h(H)=\{-2,-1,0,1,2\}
$$

Let us prove that

$$
\cap\{C h(f H): f \in C[-2,2], f>0\}=\{-2,0,2\} .
$$

Let $t \in\{-2,0,2\}$ and $f \in C[-2,2], f>0$. Let $\mu \in M_{+}^{1}([-2,2])$ be such that

$$
\left.\mu\right|_{f H}=\left.e_{t}\right|_{f H} .
$$

Then we have $\mu\left(f h_{3}\right)=f(t) h_{3}(t)=0$. This yields

$$
\operatorname{supp} \mu \subset\{-2,0,2\} \text {. }
$$

Hence there exist $a, b, c \in[0,1]$ such that

$$
\begin{gathered}
a+b+c=1, \\
\mu=a e_{-2}+b e_{0}+c e_{2} .
\end{gathered}
$$

From (3.15) and (3.16) we obtain

$$
\begin{cases}f(-2) a-f(2) c & =f(t) h_{1}(t) \\ -f(-2) a+f(0) b-f(2) c & =f(t) h_{2}(t) \\ a+b+c & =1\end{cases}
$$

It is easily seen that this system has a unique solution, and we deduce $\mu=e_{t}$. This means that $t \in C h(f H)$. So we have

$$
\{-2,-0,2\} \subset \cap\{C h(f H): f \in C[-2,2], f>0\} \subset C h(H)=\{-2,-1,0,1,2\} .
$$

Let us consider now the functions $f_{1}, f_{2} \in C[-2,2]$ defined by

It is easy to verify that

$$
\begin{aligned}
& f_{1}(x)= \begin{cases}\frac{1}{3}(1+2|x+1|), & x \in[-2,0], \\
1, & x \in(0,2] .\end{cases} \\
& f_{2}(x)= \begin{cases}1, & x \in[-2,0], \\
\frac{1}{3}(1+2|x-1|), & x \in(0,2] .\end{cases}
\end{aligned}
$$

$$
\begin{aligned}
& \left.\frac{1}{3}\left(e_{-2}+e_{0}+e_{1}\right)\right|_{f_{1} H}=\left.e_{-1}\right|_{f_{1} H}, \\
& \left.\frac{1}{3}\left(e_{-2}+e_{0}+e_{2}\right)\right|_{f_{2} H}=\left.e_{1}\right|_{f_{2} H} .
\end{aligned}
$$

This means that $-1 \notin C h\left(f_{1} H\right), 1 \notin C h\left(f_{2} H\right)$. Hence -1 and 1 do not belong to $\cap\{C h(f H): f \in C[-2,2], f>0\}$. From (3.17) we deduce (3.14). 


\section{References}

[1] Alfsen, E., Compact Convex Sets and Boundary Integrals, Springer 1971.

[2] Altomare, F., Campiti, M., Korovkin-type Approximation Theory and its Applications, Walter de Gruyter, Berlin, New York, 1994.

[3] Altomare, F., Cappelletti Montano, M., Leonessa, V., Raşa, I., Markov Operators, Positive Semigroups and Approximation Processes, De Gruyter Studies in Mathematics, 61, 2014.

[4] Bauer, H., Silovscher rand und Dirichletsches problem, Ann. Inst. Fourier, 11(1961), 89136.

[5] Bauer, H., Leha, G., Papadopoulou, S., Determination of Korovkin closures, Math.Z., 168(1979), 263-274.

[6] Boboc, N., Bucur, Gh., Conuri Convexe de Funcţii Continue pe Spaţii Compacte, Ed. Acad. RSR, Bucureşti, 1976.

[7] Bunce, U.W., Zame, W.R., Boundaries for subspaces of $C(K)$, Quart. J. Math. Oxford, 25(1974), 227-234.

[8] Hervé, M., Sur les representations intégrales a l'aide des points extremaux dans un ensemble compact convexe metrisable, C.R. Acad. Sci, Paris, 253(1961), 366-368.

[9] Micchelli, C.A., Convergence of positive linear operators on $C(X)$, J. Approx. Theory, 13(1975), 305-315.

[10] Phelps, R.R., Lectures on Choquet's theorem, Lecture Notes in Mathematics 1757, Springer-Verlag Berlin Heidelberg, 2001.

[11] Raşa, I., On some results of C.A. Micchelli, Anal. Numér. Théor. Approx., 9(1980), 125-127.

[12] Raşa, I., Sets on which concave functions are affine and Korovkin closures, Anal. Numér. Théor. Approx., 15(1986), no. 2, 163-165.

[13] Rogalski, M., Espaces de Banach réticulés et probléme de Dirichlet, Publ. Math. D'Orsay, Mathématique, 425, 1968-1969.

[14] Watanabe, H., The Choquet boundary and the integral representation, Nat. Sci. Rep. Ochanomizu Univ., 31(1980), 23-33.

Laura Hodiş

Technical University of Cluj-Napoca

Department of Mathematics

28, Memorandumului Street

400114 Cluj-Napoca, Romania

e-mail: mesaros.laura@gmail.com

Alexandra Măduţa

Technical University of Cluj-Napoca

Department of Mathematics

28, Memorandumului Street

400114 Cluj-Napoca, Romania

e-mail: alexandra91@yahoo.com 